\title{
Tackling malnutrition in the community, by review of the hot delivered meals service
}

\author{
R. A. Skinner ${ }^{1}$, C. Dugdale ${ }^{2}$, C. Crowe ${ }^{2}$, D. Fenlon ${ }^{2}$, J. Flaherty ${ }^{2}$ and A. Fletcher ${ }^{2}$ \\ ${ }^{1}$ Newcastle Nutrition, Royal Victoria Infirmary, Newcastle upon Tyne NE1 4LP, UK (part of Newcastle upon Tyne Hospitals \\ NHS Foundation Trust) and ${ }^{2}$ Adult and Culture Services Directorate, Civic Centre, Barras Bridge, Newcastle upon Tyne \\ NE1 8PA, UK
}

The prevalence of malnutrition is of ongoing concern on admission to institutional care settings and increases with age in those over 65 years ${ }^{(1)}$. To address this problem recommendations are now focused on improving the nutritional care of vulnerable groups in the community $^{(2)}$. Newcastle upon Tyne City Council provides a hot delivered meal service for people who are unable to safely prepare food themselves. Limited access to food is considered a pre-cursor for malnutrition. Awareness of this led a working party reviewing this service to ask the question: are people receiving hot delivered meals at nutritional risk? To answer this question and help guide the working party a survey of people receiving the service was undertaken and menus nutritionally analysed. Of 262 people receiving the meals, $118(45 \%)$ agreed to be interviewed, $69 \%$ were over 80 years of age. Nutrition screening was completed on 111 participants using the Malnutrition Universal Screening Tool 'MUST'(3).

\begin{tabular}{lc}
\hline Malnutrition risk category (MUST score) & Percentage of people \\
\hline High risk (score 2 or more) & $15 \%(n=17)$ \\
Medium risk (score of 1) & $16 \%(n=18)$ \\
Low risk (score of 0$)$ & $69 \%(n=76)$ \\
\hline
\end{tabular}

The results of the nutritional analysis were compared to current nutrient based guidelines for older people receiving community meals ${ }^{(4)}$. A number of key nutrients were met by the menu; however, average energy provided was $584 \mathrm{kcal}$ per meal, almost $20 \%$ below the recommendation for a woman. The recommendation for Folate was also not achieved.

Almost 1 in 3 of the adults surveyed was shown to be at risk of malnutrition. This supports the findings of BAPEN that malnutrition originates in the community ${ }^{(1)}$. Ensuring the main meal provided, to this vulnerable group, meets nutrient targets is essential to tackle this.

Newcastle City Council is committed to the government's nutrition action plan ${ }^{(2)}$, which aims to improve the health and wellbeing of older people through good nutritional care. The findings of the survey and analysis helped empower the working party to push forward with service improvements: The nutritional content of the menu has been improved. Meal delivery drivers have been trained on the importance of good nutrition, spotting signs of malnutrition and providing support to facilitate uptake of the meal. All adults requiring care services including hot delivered meals are now nutritionally screened on referral, ensuring those at risk are identified quickly.

1. British Association for Parenteral and Enteral Nutrition BAPEN (2009) Nutrition Screening Survey in the UK in 2008. Redditch, Worcs.: BAPEN.

2. Department of Health (2007) Improving Nutritional Care: A Joint Action Plan from the Department of Health and Nutrition Summit Stakeholder Group. Leeds: Department of Health.

3. British Association for Parenteral and Enteral Nutrition BAPEN (2003) The 'MUST' Report: Nutritional Screening of Adults - A Multidisciplinary Responsibility. Redditch, Worcs: BAPEN.

4. The Caroline Walker Trust (1995) Eating Well for Older People: Practical and Nutritional Guidelines for Food in Residential and Nursing Homes and for Community Meals, 2nd ed. St Austell: The Caroline Walker Trust. 